\title{
Status of the CUORE and CUORE-0 Experiments at Gran Sasso
}

Jonathan Ouellet ${ }^{* \dagger}$

University of California, Berkeley

E-mail: jlouellet@lbl.gov

CUORE is a $741 \mathrm{~kg}$ array of $\mathrm{TeO}_{2}$ bolometers that will search for the neutrinoless double beta decay of ${ }^{130} \mathrm{Te}$. The detector is being constructed at the Laboratori Nazionali del Gran Sasso in Italy, where it will start taking data in 2015. If the target background of 0.01 counts $/(\mathrm{keV} \cdot \mathrm{kg} \cdot \mathrm{y})$ is reached, then we expect that in five years of data taking CUORE will have an half life sensitivity of about $10^{26} \mathrm{y}$. CUORE- 0 is a smaller experiment constructed to test and demonstrate the performance expected for CUORE. The detector is a single CUORE-like tower of 52 bolometers that started taking data in March 2013. Here, the status and perspectives of CUORE is discussed, and the second CUORE-0 data release is presented.

Technology and Instrumentation in Particle Physics 2014,

2-6 June, 2014

Amsterdam, the Netherlands

* Speaker.

†n behalf of the CUORE collaboration 


\section{Neutrinoless Double Beta Decay}

Currently, there are many open questions about the nature of the neutrino. What is the mass hierarchy? What is the absolute mass scale of the neutrino? Is the neutrino mass a Dirac type mass as with the other fermions, or is the neutrino unique in having a Majorana type mass? Finally, why is the mass scale so different from all the other fermions?

Neutrinoless double beta decay $(0 v \beta \beta),(Z, A) \rightarrow(Z+2, A)+2 \beta$, is an as-yet undiscovered process that could provide many insights into the fundamental nature of the neutrino as well as the symmetries of the lepton sector as a whole. Its discovery would indicate a violation of lepton number and can only occur if the neutrino has a Majorana mass term. In fact, it is currently the most realistic mechanism for addressing the Majorana/Dirac nature of the neutrino mass $[1,2]$. Combined with other experiments, neutrinoless double beta decay could also provide constraints to the neutrino mass hierarchy and scale.

In the simplest model of $0 v \beta \beta$, moderated by light Majorana neutrinos, the decay rate is given by

$$
\Gamma=G^{0 v}\left|M^{0 v}\right|^{2} \frac{\left|\left\langle m_{\beta \beta}\right\rangle\right|^{2}}{m_{e}^{2}}
$$

where $G^{0 v}$ is a phase space factor, $M^{0 v}$ the nuclear matrix element, $\left\langle m_{\beta \beta}\right\rangle$ is the effective Majorana neutrino mass, and $m_{e}$ is the electron mass for normalization [1]. The phase space factor is accurately calculable and is proportional to the $5^{\text {th }}$ power of the total decay energy [3]. The matrix element is the largest source of theoretical uncertainty and different models disagree by factors of up to 2 or 3 . This uncertainty induces a large spread in the limits placed on $\left\langle m_{\beta \beta}\right\rangle$ $[4,5,6,7,8,9,10]$. The current generation of $0 v \beta \beta$ experiments, running or under construction, are generally sensitive to $\left\langle m_{\beta \beta}\right\rangle$ in the range 50 to $200 \mathrm{meV}$.

Two neutrino double beta decay $(2 v \beta \beta),(Z, A) \rightarrow(Z+2, A)+2 \beta+2 \bar{v}_{e}$, which is allowed in the Standard Model, has been observed in a handful of isotopes. Since detectors don't detect the energy of the neutrinos, the $2 v \beta \beta$ spectrum forms a continuous spectrum from zero energy to the full energy of the decay. But since $0 v \beta \beta$ produces no neutrinos, its energy spectrum is not similarly broadened. The experimental signature of $0 v \beta \beta$ would be a narrow peak at the full energy of the decay, broadened only by the energy resolution of the detector.

The sensitivity to this process can be modeled as a single bin counting experiment. In the limit of gaussian statistics, a common relation between the detector parameters and the half-life sensitivity is

$$
T_{1 / 2}^{0 v} \propto \eta \cdot a \times \sqrt{\frac{M \cdot t}{b \cdot \Delta E}}
$$

where $\eta$ is the detector efficiency, $a$ is the isotopic abundance, $M$ is the total active mass, $t$ is the live time of the experiment, $\Delta E$ is the energy resolution at the region of interest (ROI), and $b$ is the background rate per unit energy and mass [11].

The CUORE experiment searches for the $0 v \beta \beta$ of the isotope ${ }^{130} \mathrm{Te}$. This isotope is an attractive candidate for at least three main reasons. First, the single beta decay channel is kinematically 
forbidden, so the nucleus can only decay via the higher order double beta decay channel. Second, ${ }^{130}$ Te has a high natural isotopic abundance (34.4\%) [12] and so does not require isotopic enrichment. And finally, its relatively high decay energy, $2528 \mathrm{keV}$, puts the region of interest above the majority of the naturally occurring $\gamma$ background.

The predecessor to CUORE, called Cuoricino, ran from 2003-2008 in Hall A of the Laboratori Nazionali del Gran Sasso (LNGS) in Italy. With an exposure of $19.8 \mathrm{~kg} \cdot \mathrm{yr}$ of ${ }^{130} \mathrm{Te}$, Cuoricino set the current best limit on the ${ }^{130} \mathrm{Te} 0 v \beta \beta$ of $2.8 \times 10^{24} \mathrm{yr}(90 \%$ C.L.).

In this proceeding, we briefly review the working principle of the CUORE detector and present the present status of the detector construction. In section 3, we present the second data release from the CUORE-0 detector, including improvements made since the first data release and increased statistics. In section 4, I discuss the outlook for both CUORE-0 and its implications for CUORE.

\section{The CUORE Experiment}

\subsection{A CUORE Bolometer}

The CUORE detector is an array of 988 bolometers each operated independently as an individual search for $0 v \beta \beta$. A CUORE bolometer is composed of two main components, the absorber which converts an energy deposition into a change in temperature and the thermistor which converts this change in temperature into a measurable change in voltage.

A CUORE absorber is a $5 \times 5 \times 5 \mathrm{~cm}^{3} \mathrm{TeO}_{2}$ crystal weighing approximately $750 \mathrm{~g}$. Since the crystal is made from ${ }^{\text {nat }} \mathrm{Te}$, it is both the source and detector of the decays of interest. The

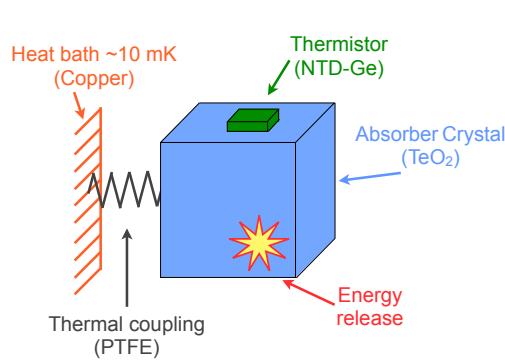

Figure 1: Schematic of a bolometer. crystals are arranged into an array of 19 towers, of 13 floors each, with 4 crystals per floor. The CUORE detector will have a total active mass of $741 \mathrm{~kg}$, for $206 \mathrm{~kg}$ of ${ }^{130} \mathrm{Te}$. The copper support frames act as the bolometers' fixed temperature heat bath. The detector is held at an operating temperature of $\sim 10 \mathrm{mK}$ in a dilution refrigerator. At this low temperature, the heat capacity of a single crystal is such that a $1 \mathrm{MeV}$ energy deposition corresponds to a change in temperature of about $100 \mu \mathrm{K}$ - about $1 \%$. This small change in temperature is read out using a Neutron Transmutation Doped Germanium thermistor (NTD). These thermistors have a resistivity which is exponentially dependent on temperature, and amplify the $\sim 1 \%$ temperature change to a change in resistivity of $\sim 10 \%$. The NTDs have typical resistances of $\sim 100 \mathrm{M} \Omega$ and are current biased and read out using room temperature electronics.

\subsection{Background Reduction Strategy}

One of the primary goals in the design and assembly of CUORE was to decrease the background rate from that seen in Cuoricino. For CUORE, we aimed for a maximum background rate of 0.01 counts $/(\mathrm{keV} \cdot \mathrm{kg} \cdot \mathrm{yr})$ at the full energy of the ${ }^{130} \mathrm{Te}$ decay. We worked closely with the $\mathrm{TeO}_{2}$ crystal grower at the Shanghai Institute of Ceramics, Chinese Academy of Science to limit both the surface and bulk contamination of the crystals themselves [13]. We measured the bulk and surface contamination to be less than $5.0 \times 10^{-7}$ and $1.3 \times 10^{-6} \mathrm{~Bq}$ per crystal, respectively, in ${ }^{238} \mathrm{U}$ and 
$6.3 \times 10^{-7}$ and $3.0 \times 10^{-7} \mathrm{~Bq}$ per crystal, respectively, in ${ }^{232} \mathrm{Th}$ (90\% C.L.) [14]. We transported the crystals from Shanghai to LNGS at sea level in order to minimize cosmogenic activation.

To reduce the amount of contamination from the supporting structure of our detector, we developed and tested strict material selection and cleaning procedures. By redesigning the tower support structure, we reduced the amount of copper needed per crystal by a factor of about $\sim 2$. To remove the contamination on the surface of the copper facing our detectors, we developed a copper treatment procedure that combines tumbling, electropolishing, chemical etching and magnetic plasma etching. The result of this treatment reduced the total surface contamination of the copper to below $1.3 \times 10^{-7} \mathrm{~Bq} / \mathrm{cm}^{2}$ at $90 \%$ C.L. in both ${ }^{238} \mathrm{U}$ and ${ }^{232} \mathrm{Th}$ [15]. To minimize re-contamination from radon in the air, we assemble and store all of the towers in glove boxes under constant $\mathrm{N}_{2}$ flux in a class 1000 clean room.

\subsection{Current Status of the CUORE Experiment}

As of this writing, the assembly and instrumentation of the entire CUORE detector is complete. The towers are being stored under constant $\mathrm{N}_{2}$ flux awaiting the completion of the CUORE cryostat. A detailed status of the cryostat can be found elsewhere in these proceedings.

\section{Second Data Release from CUORE-0}

In addition to the 19 towers produced for CUORE, a $20^{\text {th }}$ tower was produced - the first tower, in fact - and is being operated in the old Cuoricino cryostat as an independent experiment called CUORE-0. It was originally foreseen as a full scale debugging of the assembly procedure for CUORE, but is a formidable $0 v \beta \beta$ search in its own right. It has been collecting data since March 2013, and has so far amassed $18.1 \mathrm{~kg} \cdot \mathrm{yr}$ of total exposure (5.0 $\mathrm{kg} \cdot \mathrm{yr}$ of ${ }^{130} \mathrm{Te}$ exposure) after removing noisy periods of the detector. The data taking are split into two phases, Phase I ran from March 2013 - August 2013 and collected $7.1 \mathrm{~kg} \cdot \mathrm{yr}$ of total exposure, before a temporary shutdown to perform maintenance on the cryostat, and Phase II ran from November 2013 - May 2014 and collected an additional $11.0 \mathrm{~kg} \cdot \mathrm{yr}$ of total exposure. Here I present the detector performance from Phase II and the combined statistics from Phases I \& II.

\subsection{Detector Performance}

The data collection for CUORE-0 is split into datasets. Each dataset is about one month long with the first and last few days devoted to calibration. The calibration data are shared between adjacent datasets. During Phase I, CUORE-0 maintained about $43 \%$ live time efficiency, with much of the down time coming from maintenance of the 30 year old cryostat and calibration. During Phase II, we improved the live time efficiency to about $61 \%$ by improving the up-time of the cryostat and optimizing the amount of time spent collecting calibration data.

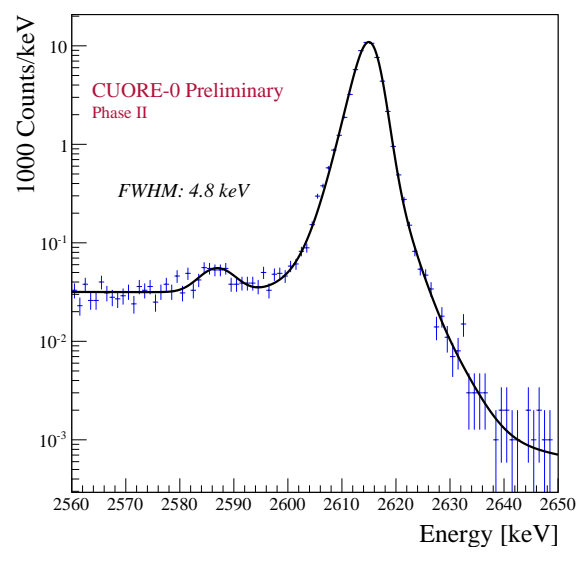

Figure 2: Fit to the ${ }^{208} \mathrm{Tl}$ photopeak in the Phase II calibration data. The FWHM of the fit is $4.8 \mathrm{keV}$. 

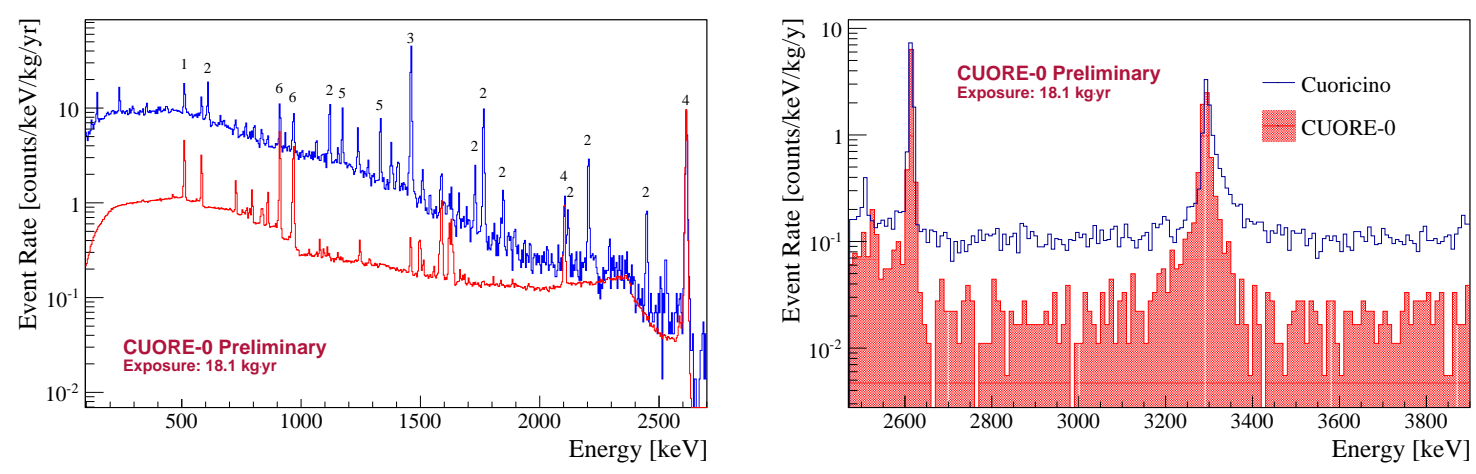

Figure 3: Left: The combined Phase I \& II calibration (red) and background (blue) spectrum from threshold to $2.7 \mathrm{MeV}$. The calibration data has been scaled to match the background ${ }^{208} \mathrm{Tl}$ peak rate. The labeled peaks are from $\gamma$ from (1) $e^{+} e^{-}$annihilation, (2) ${ }^{214} \mathrm{Bi}$, (3) ${ }^{40} \mathrm{~K}$, (4) ${ }^{208} \mathrm{Tl},(5){ }^{60} \mathrm{Co},(6){ }^{228} \mathrm{Ac}$. Right: CUORE-0 Phase I \& II combined data plotted against the Cuoricino data in the range $2.5 \mathrm{MeV}$ to $3.9 \mathrm{MeV}$. The peak at $3250 \mathrm{keV}$ is due to ${ }^{190} \mathrm{Pt}$ contamination in the crystal bulk.

We calibrate the detectors by inserting thoriated tungsten strings inside the outermost lead shielding. We use the $\gamma$ lines from the thorium daughters to determine the calibration function for each channel. The highest $\gamma$ calibration line comes from ${ }^{208} \mathrm{Tl}$ decay at $2615 \mathrm{keV}$ - only $87 \mathrm{keV}$ above from our ROI. Since the rate of the background $\gamma$ lines in our $0 v \beta \beta$ search data is very low, we use this line in the calibration data to determine our energy resolution on a channel by channel basis.

We determine our energy resolution by first fitting the ${ }^{208} \mathrm{Tl}$ line with a p.d.f. that accommodates the resolution of the channel, a low energy tail, a compton continuum, a flat background and a Te X-ray escape peak that lies $\sim 30 \mathrm{keV}$ below the main peak. We then sum the channel p.d.f.s together, weighted by the number of counts contributing and then measure the FWHM of the summed p.d.f.

For Phase I, we achieved an energy resolution of $6.3 \mathrm{keV}$ FWHM [16], while in Phase II we improved the resolution to $4.8 \mathrm{keV}$ FWHM. The majority of the improvement came from more stable and less noisy operation of the cryostat. The fit for Phase II, excludes the first dataset of the phase, since we were still adjusting the noise level. The combined Phase I \& II data, including all datasets for CUORE-0, has a resolution of $5.3 \mathrm{keV}$ FWHM. The fit to the Phase II data alone is shown in Fig. 2.

For the $0 v \beta \beta$ search data, we do not have enough statistics to study the detector resolution on a channel by channel basis, so we simply fit a purely phenomenological double gaussian to the summed data and measure the FWHM of the fit p.d.f. For the full Phase I \& II data, we measure an energy resolution of $5.2 \mathrm{keV}$ FWHM, improved from $5.9 \mathrm{keV}$ FWHM in Phase I alone. While the energy resolution in the $0 v \beta \beta$ search data is slightly better than the resolution in the calibration data, it is consistent within statistics.

\subsection{Results}

The data analysis procedures are identical to those used in Phase I and are detailed elsewhere [16]. The CUORE-0 $0 v \beta \beta$ search spectrum below the ${ }^{208} \mathrm{Tl}$ is show in Fig. 3 along with the 

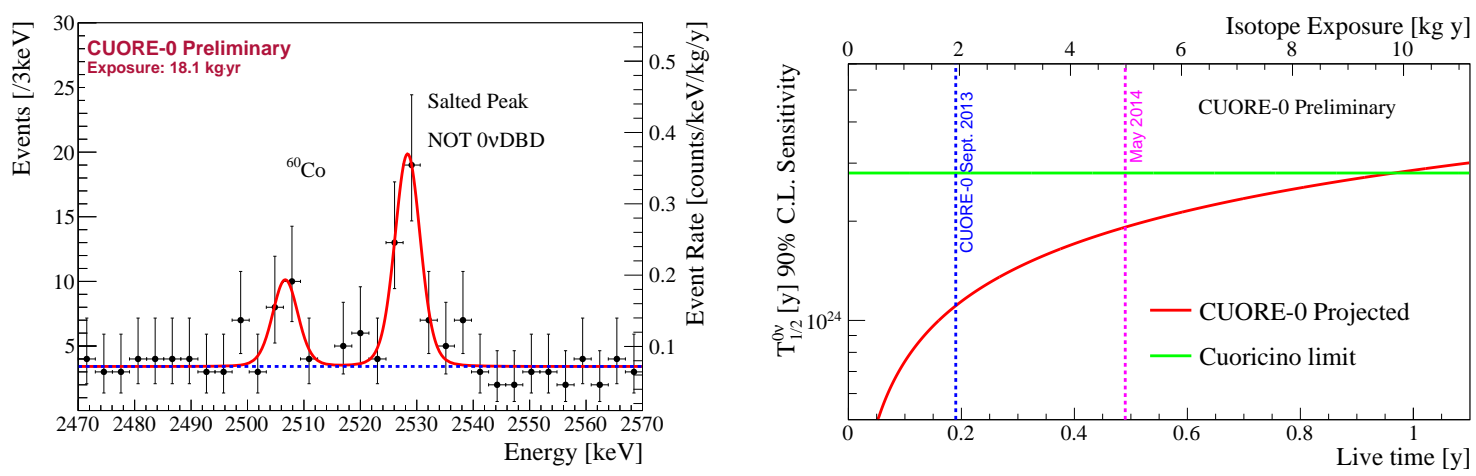

Figure 4: Left: The CUORE-0 spectrum around the ROI. The peak at $2505 \mathrm{keV}$ is due to the sum of two ${ }^{60} \mathrm{Co} \gamma$ 's, the peak in the ROI at $2528 \mathrm{keV}$ is the salted peak from our blinding procedure (i.e. not a true signal peak). Right: Sensitivity projection of CUORE-0 experiment.

calibration spectrum.

The signal efficiency for the event selection is $89.7 \pm 0.9 \%$ compared to $92.9 \pm 1.8 \%$ in Phase I alone. We combine this with the double beta decay single crystal containment efficiency which we estimate using Monte Carlo to be $87.4 \pm 1.1 \%$, and the trigger efficiency which is measured on pulser events to be $99.00 \pm 0.01 \%$. The overall signal efficiency for the Phase I \& II data is $77.6 \pm 1.3 \%$. The event selection cuts are not yet optimized, and we plan to optimize them before unblinding.

The most significant background in Cuoricino came from degraded $\alpha$ particles, which deposit only a fraction of their total energy into a crystal. These $\alpha$ events form a flat spectrum which stretch from the $\alpha$ region down through the ROI, limiting the sensitivity of the experiment. We estimate the degraded $\alpha$ background contribution by measuring the event rate in region 2.7 to 3.9 $\mathrm{MeV}$, excluding the ${ }^{190} \mathrm{Pt}$ peak between 3.2 and 3.4 MeV. In CUORE-0, we measure this rate to be $0.020 \pm 0.001$ counts $/(\mathrm{keV} \cdot \mathrm{kg} \cdot \mathrm{yr})$ as compared to $0.11 \pm 0.001 \mathrm{counts} /(\mathrm{keV} \cdot \mathrm{kg} \cdot \mathrm{yr})$ in Cuoricino.

In Fig 4, we show the blinded data in the ROI. Our blinding procedure is a form of data salting, that moves a significant and blinded number of events from the ${ }^{208} \mathrm{Tl}$ line into the ROI, masking any physical signal or fluctuation and producing an artificial — or salted — peak. The fit is a flat background with two gaussians, one for the ${ }^{60} \mathrm{Co}$ sum peak fixed at $2505 \mathrm{keV}$ and one for the salted peak fixed at $2528 \mathrm{keV}$. The gaussian widths are channel specific, and taken from the fits to the calibration data. The best fit background rate in the ROI is $0.063 \pm 0.006$ counts/(keV·kg.year).

\section{Discussion and Outlook}

\subsection{CUORE-0 Outlook}

In Phase II of data taking, we have improved the livetime efficiency of the CUORE-0 detector by about 50\%. We have also improved the noise level of the detector and achieved the goal resolution of $5 \mathrm{keV}$ FWHM.

In Fig. 4, we show the projected sensitivity of CUORE-0. We expect CUORE-0 will surpass the Cuoricino sensitivity with about 1 year of live time. However, we are still developing algorithms for the analysis chain to remove common mode noise to improve the energy resolution, 
and in parallel we are still developing and optimizing our event selection with the hope of further decreasing the background. Because of these, the CUORE-0 sensitivity may improve in the future.

\subsection{CUORE Outlook}

In CUORE-0, we see a factor of $\sim 6$ reduction in the rate of degraded $\alpha$ events. This reduction in background demonstrates the success of the background reduction techniques developed for CUORE. We expect the background rate from degraded $\alpha$ 's to be decreased further in CUORE due to the smaller amount of copper surface area per crystal. In CUORE-0, we also see a difference in background rate between the ROI and the degraded $\alpha$ region of about $0.043 \pm 0.006$ counts $/(\mathrm{keV} \cdot \mathrm{kg} \cdot \mathrm{yr})$. This difference is consistent with what was observed in Cuoricino and we attribute it to compton scattered ${ }^{208} \mathrm{Tl} \gamma$ rays originating in the cryostat materials. We expect that this background will also be significantly reduced in CUORE because of better material selection for the CUORE cryostat, improved shielding and more effective anti-coincidence rejection between multiple crystals.

We are confident that the CUORE background goal of 0.01 counts $/(\mathrm{keV} \cdot \mathrm{kg} \cdot \mathrm{yr})$ in the ROI is well within reach. With this background rate, and an energy resolution of $5 \mathrm{keV}$, CUORE will be sensitive to a ${ }^{130} \mathrm{Te} 0 v \beta \beta$ half-life of $1.6 \times 10^{26} \mathrm{yr}$ at the $90 \%$ C.L. [11] with 5 years of run time. This corresponds to a $1 \sigma$ sensitivity range in $m_{\beta \beta}$ of $41-95 \mathrm{meV}$.

\section{References}

[1] F. T. Avignone, S. R. Elliott, J. Engel, Double beta decay, Majorana neutrinos, and neutrino mass, Rev. Mod. Phys. 80 (2008) 481-516.

[2] S. M. Bilenky, C. Giunti, Neutrinoless double-beta decay. a brief review. [arXiv:1203.5250]

[3] J. Kotila, F. Iachello, Phase-space factors for double- $\beta$ decay, Phys. Rev. C 85 (2012) 034316.

[4] J. Menendez, A. Poves, E. Caurier, F. Nowacki, Disassembling the Nuclear Matrix Elements of the Neutrinoless beta beta Decay, Nucl. Phys. A 818 (2009) 139-151. [arXiv: 0801 . 3760]

[5] P. K. Rath, et al., Uncertainties in nuclear transition matrix elements for $\beta^{+} \beta^{+}$and $\varepsilon \beta^{+}$modes of neutrinoless positron double- $\beta$ decay within the projected hartree-fock-bogoliubov model, Phys. Rev. C 87 (2013) 014301.

[6] T. R. Rodriguez, G. Martinez-Pinedo, Energy density functional study of nuclear matrix elements for neutrinoless $\beta \beta$ decay, Phys. Rev. Lett. 105 (2010) 252503. [arXiv : 1008 . 5260]

[7] D.-L. Fang, A. Faessler, V. Rodin, F. Simkovic, Neutrinoless double beta decay of deformed nuclei within QRPA with realistic interaction, Phys. Rev. C 83 (2011) 034320. [arXiv: 1101.2149 ]

[8] A. Faessler, V. Rodin, F. Simkovic, Nuclear matrix elements for neutrinoless double-beta decay and double-electron capture, J. Phys. G: Nucl. Part. Phys. 39 (2012) 124006. [arXiv:1206.0464]

[9] J. Suhonen, O. Civitarese, Review of the properties of the $0 v \beta \beta$ nuclear matrix elements, J. Phys. G: Nucl. Part. Phys. 39 (2012) 124005.

[10] J. Barea, J. Kotila, F. Iachello, Nuclear matrix elements for double- $\beta$ decay, Phys. Rev. C 87 (2013) 014315. [arXiv:1301.4203]

[11] F. Alessandria, et al., Sensitivity of CUORE to Neutrinoless Double-Beta Decay, Submitted to Astroparticle Physics. [arXiv:1109.0494] 
[12] M. A. Fehr, M. Rehkamper, A. N. Halliday, Application of MC-ICPMS to the precise determination of tellurium isotope compositions in chondrites, iron meteorites and sulfides, Int. J. Mass spectrom. (2004) 83-94.

[13] C. Arnaboldi, et al., Production of high purity TeO2 single crystals for the study of neutrinoless double beta decay, J. Cryst. Growth 312 (2010) 2999. [doi : $10.1016 /$ j . jcrysgro. 2010.06 .034$]$

[14] F. Alessandria, et al., CUORE crystal validation runs: results on radioactive contamination and extrapolation to CUORE background, Astropart. Phys. 35 (2012) 839-849. [arXiv: 1108 . 4757]

[15] F. Alessandria, et al., Validation of techniques to mitigate copper surface contamination in CUORE, Astropart. Phys. 45 (2013) 13-22. [arXiv: 1210 . 1107]

[16] D. R. Artusa, et al., Initial performance of the CUORE-O experiment, Submitted to Eur. Phys. J. C. [arXiv:1402.0922] 